\title{
RESEARCH
}

Open Access

\section{Role of PCK1 gene on oil tea-induced glucose homeostasis and type 2 diabetes: an animal experiment and a case-control study}

Qiantu Hu${ }^{1 \dagger}$, Huafeng Chen ${ }^{2+}$, Yanli $\mathrm{Zuo}^{3+}$, Qin He${ }^{2}$, Xuan He${ }^{2}$, Steve Simpson $\mathrm{Jr}^{4,5}$, Wei Huang ${ }^{2}$, Hui Yang ${ }^{2}$, Haiying Zhang ${ }^{1,6^{*}}$ and Rui Lin ${ }^{1,2,6^{*}}$ (D)

\begin{abstract}
Background: Oil tea is a type of traditional tea beverage used for treating various ailments in minority population in Guangxi, China. Our previous study showed oil tea improved glucose and lipid levels in type 2 diabetic mice. Yet, the underling molecular mechanisms are still not understood. This study aimed at assessing the effect of oil tea on glucose homeostasis and elucidating the molecular mechanisms underlying the oil tea-induced antidiabetic effects.

Methods: Twenty seven $\mathrm{db} / \mathrm{db}$ mice were gavaged with saline, metformin and oil tea for 8 weeks with measurement of biochemical profiles. A real-time ${ }^{2}\left(R^{2}\right)$ profiler polymerase chain reaction (PCR) array comprising 84 genes involved in glucose metabolism was measured and validated by quantitative PCR (qPCR). The association between the candidate genes and type 2 diabetes were further analyzed in a case-control study in the Chinese minority population.

Results: Oil tea treatment facilitated glucose homeostasis by decreasing fasting blood glucose and total cholesterol, and improving glucose tolerance. Suppressing phosphoenolpyruvate carboxykinase 1 (PCK1) expression was observed in the oil tea treatment group and the expression was significantly correlated with fasting blood glucose levels. Target prediction and functional annotation by WEB-based GEne SeT AnaLysis Toolkit (WebGestalt) revealed that $P C K 1$ mainly involved in the glycolysis/gluconeogenesis pathway among the top Kyoto Encyclopedia of Genes and Genomes (KEGG) database pathways. Both rs707555 and rs2071023 in PCK1 were significantly associated with type 2 diabetes in the minority population of Guangxi.
\end{abstract}

Conclusion: Our findings indicated oil tea improved glucose homeostasis via down-regulation of PCK1 and PCK1 may be a genetic marker for the treatment of type 2 diabetes.

Keywords: Fasting blood glucose, Glucose tolerance, Glycolysis/gluconeogenesis pathway, Oil tea, $P C K 1, R^{2}$ profiler PCR array, SNP, Type 2 diabetes

\footnotetext{
* Correspondence: zhanghaiying@gxmu.edu.cn; linrui@gxmu.edu.cn

${ }^{\dagger}$ Qiantu Hu, Huafeng Chen and Yanli Zuo contributed equally to this work.

${ }^{1}$ Center for Genomic and Personalized Medicine, Guangxi Medical University,

22 Shuangyong Road, Nanning 530021, Guangxi, China

Full list of author information is available at the end of the article
}

(c) The Author(s). 2019 Open Access This article is distributed under the terms of the Creative Commons Attribution 4.0 International License (http://creativecommons.org/licenses/by/4.0/), which permits unrestricted use, distribution, and reproduction in any medium, provided you give appropriate credit to the original author(s) and the source, provide a link to the Creative Commons license, and indicate if changes were made. The Creative Commons Public Domain Dedication waiver (http://creativecommons.org/publicdomain/zero/1.0/) applies to the data made available in this article, unless otherwise stated. 


\section{Background}

Oil tea is a unique beverage and an important dietary component in the minority areas of Guangxi Province, China. Oil tea is rich in polyphenol, caffeine, 6-gingerol and several minerals [1], and is the most popular traditional beverage used for treating various ailments in Guangxi [2]. Green tea and ginger, as the main ingredients of oil tea, individually have exhibited various biological activities, including anticancer effects, antioxidative effects, and antidiabetic effects [3-5]. Previously, our study showed oil tea treatment improved hyperglycemia, glucose intolerance and hyperlipidemia in $\mathrm{db} / \mathrm{db}$ mice [1]. While the underlying molecular mechanisms of the beneficial effects of oil tea was unknown.

Phosphoenolpyruvate carboxykinase $1(P C K 1)$ is a key gluconeogenic enzyme in the liver [6]. It is the cytosolic form of phosphoenolpyruvate carboxykinase (PCK or PEPCK) and catalyzes the initial step in hepatic gluconeogenesis. The expression of PCK1 gene is regulated by insulin, glucocorticoids, glucagon, cyclic adenosine monophosphate (cAMP) and diet, all of which have been considered associated with diabetic conditions. It has been reported that $P C K 1$ is up-regulated in diabetic rodent models [7], and overexpression in the transgenic mice resulted in insulin resistance [8] and hyperglycemia [9]. Reciprocally, silencing of PCK1 has been demonstrated to improve glycemia control, insulin sensitivity and dyslipidemia in $\mathrm{db} / \mathrm{db}$ mice [10]. Furthermore, research has indicated that induced expression of PCK1 may be a useful indicator for effect assessment in diabetes therapy. Indeed, studies of the effect of Rosmarinic acid, insulin, retinoic acid and phenobarbital showed decreased expression of PCK1 and improvement of hyperglycemia in diabetic rats [11-13]. Moreover, PCK1 has been demonstrated to be a candidate genetic marker for diabetes and obesity risk [14], and more recent research implicated this locus in type 2 diabetes (T2D) risk in Chinese [15], South Asian [16] and Finnish populations [17].

In the present study, we investigated the molecular mechanisms of antidiabetic effect of oil tea by examining the expression of $P C K 1$, as well as other genes involved in glucose metabolism in $\mathrm{db} / \mathrm{db}$ mice. We also assessed whether PCK1 is a genetic marker for T2D risk in the minority population in Guangxi.

\section{Methods}

\section{Preparation of oil tea}

The oil tea was prepared as previously described $[1,18]$. In brief, the proportions of green tea, ginger, peanut, cooking oil and salt were 9:10:5:2:1. The manufacturing process was as follows: firstly, soaked green tea leaf was stir-fried and beaten with ginger, peanut and cooking oil in a hot iron pot for $3 \mathrm{~min}$, then hot water and salt added, and the mixture boiled for $1 \mathrm{~min}$ and the resulting decoction retained. This process was repeated twice, whereupon the mixed decoction was called oil tea. The oil tea decoction then was concentrated by a rotary vacuum evaporator (IKA Works, Germany) and stored at $-80{ }^{\circ} \mathrm{C}$ until use.

\section{Animal model and experimental design}

The experimental procedures were approved by the Animal Ethics Committee of Guangxi Center for Disease Prevention and Control (Approval No. 20160003). Six week-old male diabetic $\mathrm{C} 57 \mathrm{BL} / \mathrm{Ks}$ - $\mathrm{db} / \mathrm{db}(\mathrm{db} / \mathrm{db})$ mice were randomly divided assigned to three groups and orally gavaged with oil tea $(4 \mathrm{~g} / \mathrm{kg}$ body weight $(\mathrm{BW}) /$ day, $n=10)$, distilled water $(0.02 \mathrm{ml} / \mathrm{g} \mathrm{BW} /$ day, $n=7)$ and metformin (MET, Sigma, USA, the first-line medication for treating T2D, $150 \mathrm{mg} / \mathrm{kg} \mathrm{BW} /$ day, $n=10$ ) for 8 weeks. The oil tea contained $24.6 \%$ polyphenols and $0.3 \%$ [6]-gingerol, as described previously [1]. Up to five mice per cage were maintained in a temperature-controlled $\left(22 \pm 2{ }^{\circ} \mathrm{C}\right)$ mouse facility on a reverse 12-h light/dark cycle and provided water and chow-diet ad libitum. The full nutritional composition of oil tea has been described previously [1]. Body weight and food intake were measured each week.

\section{FBG detection and OGTT test}

Fasting blood glucose (FBG) levels were examined every week after the $\mathrm{db} / \mathrm{db}$ mice overnight fasting. The oral glucose tolerance test (OGTT) was performed at weeks 4 and 8 after the mice were orally gavaged with oil tea or MET, combined with glucose solution $(1 \mathrm{~g} / \mathrm{kg} \mathrm{BW})$. Blood glucose levels were measured in tail blood samples collected at 0, 15, 30, 60, 90 and $120 \mathrm{~min}$ after glucose treatment. Blood glucose was measured using a glucometer (OMRON Healthcare Co., Ltd., China).

\section{Serum lipid measurement}

At the end of experiment, mice were killed by cervical dislocation, and blood samples were collected for the measurement of biochemical profiles. Levels of serum triglycerides (TG), total cholesterol (TC), low-density lipoprotein (LDL) and high-density lipoprotein (HDL) were measured using commercial kits (Shanghai Enzyme-linked Biotechnology Co., Ltd., China). Insulin was measured using a commercial Mouse Insulin ELISA kit (ALPCO Diagnostics, USA).

\section{$\mathrm{RT}^{2}$ profiler $\mathrm{PCR}$ array analysis}

After mice were killed, the fresh livers were collected and snap frozen in liquid nitrogen, and then stored at $80^{\circ} \mathrm{C}$ until analysis. Total RNA was isolated from the liver samples of $\mathrm{db} / \mathrm{db}$ control group and oil tea group using Qiagen RNeasy ${ }^{\circ}$ Mini Kit (QIAGEN, Shanghai, 
China) according to the manufacturer's instructions. Single-strand cDNA was synthesized from $1 \mu \mathrm{g}$ of total RNA by reverse transcription reaction using Qiagen $\mathrm{RT}^{2}$ First Strand Kit (QIAGEN, Shanghai, China). The cDNA was mixed with Qiagen PCR $\mathrm{RT}^{2}$ SYBR Green Master Mix (QIAGEN, Shanghai, China).

To explore the underlying mechanisms of oil tea induced-effects, the expression of 84 genes involved in glucose metabolism including PCK1 were examined using a Qiagen mouse glucose metabolism $\mathrm{RT}^{2}$ profiler PCR array (PAMM-006Z, QIAGEN, Shanghai, China). Relative quantification of mRNA levels was determined by real-time quantitative PCR using a Bio-Rad CFX96 Sequence Detector instrument. The quantitative expression of gene was calculated from the cycle threshold (CT) value of each sample in the linear part of the curve using the relative quantification method $\left(2^{-\Delta \Delta C T}\right)$ [19]. The samples were analyzed in triplicate and corrected for the selected internal standard which had the smallest standard deviation among the housekeeping genes. Candidate genes were selected from those whose expressions differed greater than 1.25 -fold or less than 0.75 -fold, or which differed significantly $(p<0.05)$ between the control group and oil tea treatment group.

\section{Quantitative PCR (qPCR) analysis for the candidate genes}

For those candidate genes selected from the $\mathrm{RT}^{2}$ profiler PCR array, specific PCR primers were designed for further quantitative real-time PCR analysis (Takara, Dalian, China) as follows.

\section{ALDOA: 5' GCTCCTTAGTCCTTTCGCCT - 3' and 5- TCAGTGCTGGGTATGGGTG - 3 \\ FBP2: 5' - GATCTGTTCATGCTGGACCC - 3 and 5- TACTTGGCATAGCCCTCGTT - 3 \\ IDH2: 5' - CAGCACTGACTGTCCCCAG - 3' and 5- CACCGTCCATCTCCACTACC - 3' \\ OGDH: 5' TGATGATGCTCCGGTAACTG - 3 and 5- AAGTGGTGGTGGGTAAGTGG - 3 \\ PCK1: 5' CTGGATGAAGTTTGATGCCC - 3 and 5- TGTCTTCACTGAGGTGCCAG - 3 \\ PCK2: 5' GTACTGGGAAGGCATTGACC - 3' and 5- AGTTTGGATGTGCACAGGGT - 3' \\ PDK2: 5'- ACGTCATTCACTTTCCTCCG - 3' and 5- TGGACATACCAGCTCTGCAC - 3' \\ PDK4: 5' - AGTGAACACTCCTTCGGTGC - 3' and 5' TGACAGGGCTTTCTGGTCTT - 3' \\ RBKS: 5' - GTAGTGGTGGGTTCCTGCAT - 3 and 5- CCTTTTCCTCCAAAGCCAA - 3 ' \\ UGP2: 5' - CAAGAAAAGGGACCGTCTGT - 3 and 5- GTTATCAGGCAAGCCTCTGG - 3'}

cDNA was synthesized with $1 \mu \mathrm{g}$ RNA using PrimeScript $^{\mathrm{tm}}$ RT reagent Kit with gDNA Eraser (Perfect Real Time, Takara, Dalian, China)and mixed with $\mathrm{SYBR}^{\circ}$ Premix Ex Taq ${ }^{\text {Tw }}$ II (Tli RNaseH Plus, Takara, Dalian, China) according to the manufacturer's instructions. The quantitative expressions of genes, again, were calculated from the CT value using the $2^{-\Delta \Delta C T}$ method. Those genes with expressions less than 0.75 -fold were selected further for pathway and biological process analysis by using WEB-based GEne SeT AnaLysis Toolkit (WebGestalt, http://www.webgestalt.org/). Target genes were those expression significantly differed between the oil tea group and $\mathrm{db} / \mathrm{db}$ control group $(p<0.05)$ and were analyzed the correlation with FBG further.

\section{Selection of the human subjects}

Eighty-six T2D patients and 286 healthy subjects were recruited from the nutrition survey conducted in the minority areas in Gongcheng Yao Autonomous County and Binyang County, Guangxi, China from 2014 to 2015. All subjects were conducted with FBG or OGTT test. T2D patients were selected based on the 1999 World Health Organization diagnostic criteria [20]: FBG $\geq 7.0 \mathrm{mmol} / \mathrm{L}$ or OGTT $\geq 11.1 \mathrm{mmol} / \mathrm{L}$ or had self-reported diagnosed T2D. Controls were the healthy subjects with normal glucose level: $\mathrm{FBG}<6.1 \mathrm{mmol} / \mathrm{L}$ and OGTT $<7.8 \mathrm{mmol} / \mathrm{L}$ without T2D history. A standard questionnaire was administered by trained staff to obtain information on demographic characteristics, personal and family medical history, etc. This study was approved by the Ethics Committee of Guangxi Center for Disease Prevention and Control (No. GXIRB2016-0014). All subjects provided informed consent.

\section{Biochemical measurements and genotyping}

Overnight fasting blood samples were obtained from cases and controls for biochemical measurement and genotyping. Participants without diagnosed diabetes were given a standard 75-g OGTT with plasma glucose measurement. Plasma glucose of FBG and OGTT were measured using the hexokinase enzymatic method, and serum triglyceride HDL-cholesterol and LDL-cholesterol were assessed using commercially available reagents (Shanghai Kehua Bio-engineering Co., Ltd., China) on an automatic analyzer (Hitachi 7080; Hitachi, Tokyo, Japan). DNA was isolated using the Thermo DNA Blood Kit (ThermoFisher Scientific (China) Co., Ltd., Guangzhou, China). Three polymorphisms (rs707555, rs2071023 and rs2070755) in PCK1 were selected based on previous research showing them to be significantly associated with T2D [15-17]. SNPs genotyping used the MassARRAY Genotyping System (Sequenom, BGI Tech., Wuhan, China). The primers were designed using MassARRAY Assay Design 3.0 software. The call rate of the genotyping SNPs achieved 99\% or higher.

\section{Statistical analyses}

Data were expressed as means $\pm \mathrm{SD}$. Comparisons between two groups were performed with two-tailed $\mathrm{t}$-test 
or Wilcoxon rank-sum test, as appropriate. Datasets that involved more than two groups were assessed by one-way ANOVA followed by Newman-Keuls post-hoc tests. The area under the curve (AUC) of OGTT was calculated by the trapezoid rule [21]. Hardy-Weinberg equilibrium (HWE) was analyzed by using the PLINK [22] software. Logistic regression was used to examine the association between the SNPs and T2D in the case-control analysis. $P$-values less than 0.05 were considered statistically significant. STATA version 12.0 (StataCorp LP, USA) was used for statistical analysis.

\section{Results}

\section{Oil tea decreases fasting and postprandial glucose}

During the 8-week experiment, lower FBG levels were observed in both oil tea and MET treatment group, and a significant decrease was observed from weeks 5 to 8 in comparison with $\mathrm{db} / \mathrm{db}$ control group $(p<0.05)$. At the end of the experiment, the FBG level of the oil tea group was significantly decreased to $15.68 \pm 3.98 \mathrm{mmol} / \mathrm{L}$, compared with the $\mathrm{db} / \mathrm{db}$ control group of $25.38 \pm 8.24$ $\mathrm{mmol} / \mathrm{L}(p<0.01)$. (Fig. 1a).

In the OGTT test, lower glucose levels were observed in both the oil tea and MET treatment groups at weeks 4 and 8 , compared to the control group. In particular, significantly lower glucose levels were observed between $60 \mathrm{~min}$ and $90 \mathrm{~min}$ in the two groups, as well as at 120 min in the MET group $(p<0.05)$. However, no significant differences were observed between oil tea group and control group at $120 \mathrm{~min}$, either at week $4(p=0.13)$ or week $8(p=0.30)$. (Fig. $1 \mathrm{~b}$ and $\mathrm{c})$. As for AUC, significantly lower levels were observed in both the oil tea and MET treatment groups at weeks 4 and $8(p<0.05$, Fig. $1 d)$.

\section{Oil tea lowers biochemical profiles}

As shown in Fig. 2a-e, significantly lower TC levels $(p<0.01)$ and a near-significantly lower TG level $(p=$ $0.06)$ were observed in the oil tea treatment group compared with the control group. Meanwhile, lower levels of LDL $(204.28 \pm 90.14$ vs $263.69 \pm 110.40 \mathrm{mmol} / \mathrm{L})$, HDL $\quad(75.71 \pm 18.16$ vs $79.01 \pm 17.49 \mathrm{mmol} / \mathrm{L})$ and insulin $(3.66 \pm 3.68$ vs $4.17 \pm 4.86 \mathrm{mmol} / \mathrm{L})$ were observed in the oil tea treatment group, although these differences did not reach significance $(p>0.05)$. However, no significant difference was observed between the MET and control groups on any lipid profile $(p>0.05)$.

\section{No change on food intake and body weight gain with oil tea treatment}

When the mice were treated with oil tea, overall there were no significant changes along the experiment except significantly lower food intake were observed at weeks 3 $(5.21 \pm 0.69$ vs $6.87 \pm 1.00 \mathrm{~g}, p<0.01)$ and $5(4.59 \pm 0.83$ vs $5.31 \pm 1.57 \mathrm{~g}, p<0.05)$. When the mice were treated with MET, the food intake was significantly lower at the first week $(2.76 \pm 2.02$ vs $5.32 \pm 1.32 \mathrm{~g}, p<0.05)$, while no significantly difference was observed at later time points
A
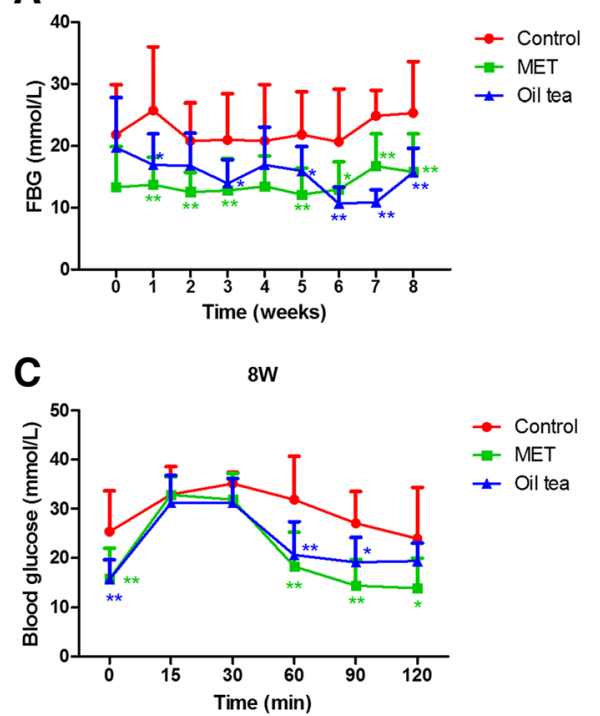

B

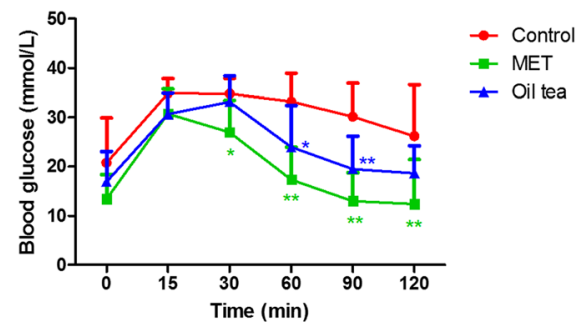

D

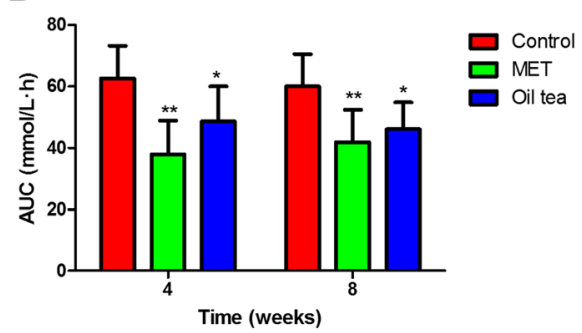

Fig. 1 Effect of oil tea on blood glucose in db/db mice. a Changes of fasting blood glucose during the 8-week experiment. b The glucose levels of the oral glucose tolerance test (OGTT) at week 4. c The glucose levels of OGTT at week 8. $\mathbf{d}$ The AUC levels of OGTT at weeks 4 and 8 . FBG, fasting blood glucose; MET, metformin; AUC, area under the curve; control group ( $n=7)$; MET group $(n=10)$; oil tea group $(n=10)$. Values are presented as means \pm SD. Differences were assessed by one-way ANOVA followed by Newman-Keuls post-hoc tests. *Significantly different from the control group $\left({ }^{*} p<0.05,{ }^{* *} p<0.01\right)$ 
A



C

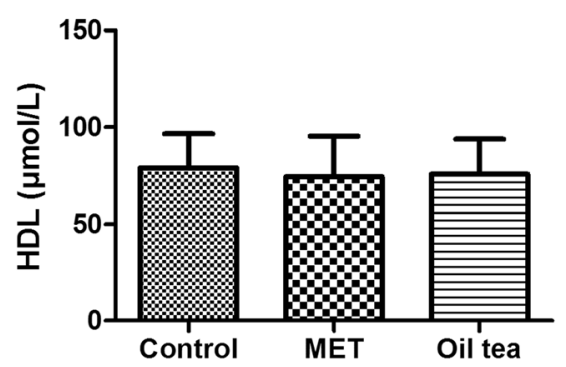

E

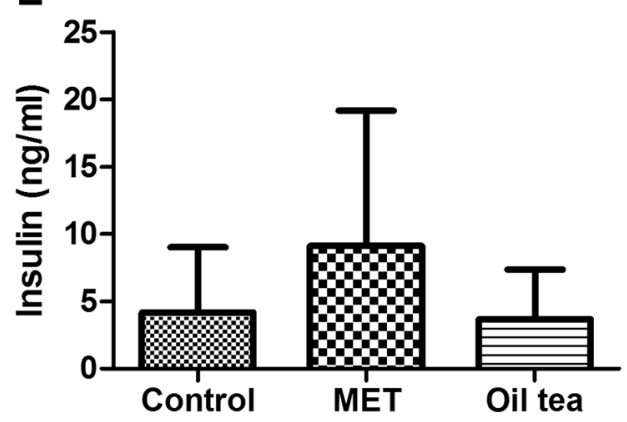

Fig. 2 Effect on serum lipid in db/db mice treated with oil tea after 8 weeks. a Effect on TC treated with oil tea. b Effect on TG treated with oil tea. $\mathbf{c}$ Effect on LDL treated with oil tea. $\mathbf{d}$ Effect on HDL treated with oil tea. e Effect on insulin treated with oil tea. TC, total cholesterol; TG, triglycerides; LDL, low-density lipoprotein; HDL, high-density lipoprotein; MET, metformin; control group ( $n=7$ ); MET group ( $n=10$ ); oil tea group $(n=10)$. Values are presented as means \pm SD. Differences were assessed by one-way ANOVA followed by Newman-Keuls post-hoc tests. *Significantly different from the control group $\left({ }^{*} p<0.05,{ }^{* *} p<0.01\right)$

(data not shown). Notably, body weight increased during the experiment in all three groups. No significant differences in weight gain were observed between the oil tea and control groups, but a significantly greater increase was observed in the MET group at weeks 7 and $8(p<0.05)$ (Fig. 3).

Oil tea decreases the expression of those genes involved in glucose metabolism pathway

Ten genes involved in the glucose metabolism were detected with different expressions between the oil tea treatment mice and control mice. Out of the 10 genes assessed, 9 genes' fold change was less than 0.75 -fold, and the expression of UGP2 (fold change $=0.76$ ) was

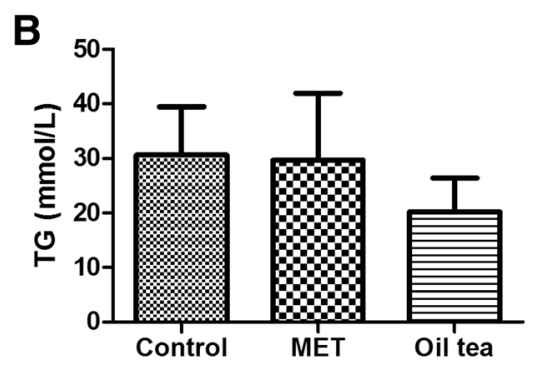

D

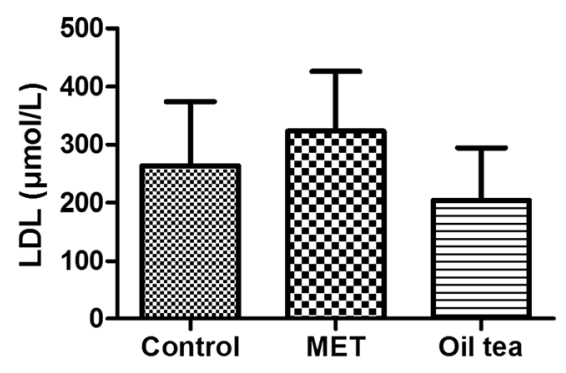




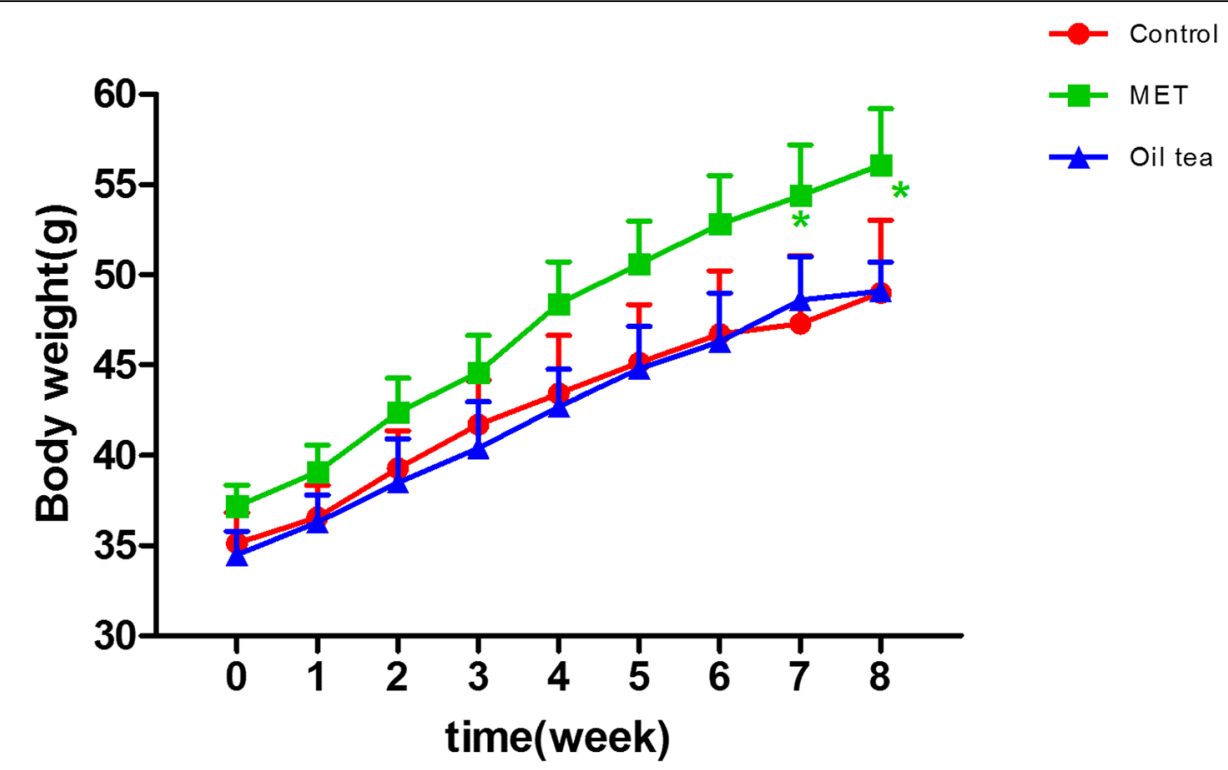

Fig. 3 Weekly body weight of mice. MET, metformin; control group $(n=7)$; MET group $(n=10)$; oil tea group $(n=10)$. Values are presented as means \pm SD. Differences were assessed by one-way ANOVA followed by Newman-Keuls post-hoc tests. *Significantly different from the control group $\left({ }^{*} p<0.05,{ }^{* *} p<0.01\right)$

\section{Association between PCK1 expression and FBG}

We next examined the association between PCK1 expression and FBG levels. We found that increased PCK1 expression was significantly correlated with increasing fasting glucose $\left(R^{2}=0.234, P<0.05\right.$, Fig. $\left.5 b\right)$. However, the expression of other four candidate genes $(A L D O A$, $F B P 2, P C K 2$ and $P D K 4$ ) were not significantly associated with fasting glucose level (data not shown).
Prediction and functional annotation by WebGestalt on PCK1 By using WebGestalt, the functional annotation results showed that $P C K 1$ in company with other four candidate genes (PCK2, ALDOA, FBP2, and PDK4) were mainly involved in glycolysis/gluconeogenesis, AMPK and insulin signaling pathway (Fig. 6a). Analysis on function, biological process showed PCK1 and the other four candidate genes to be involved in ten types of

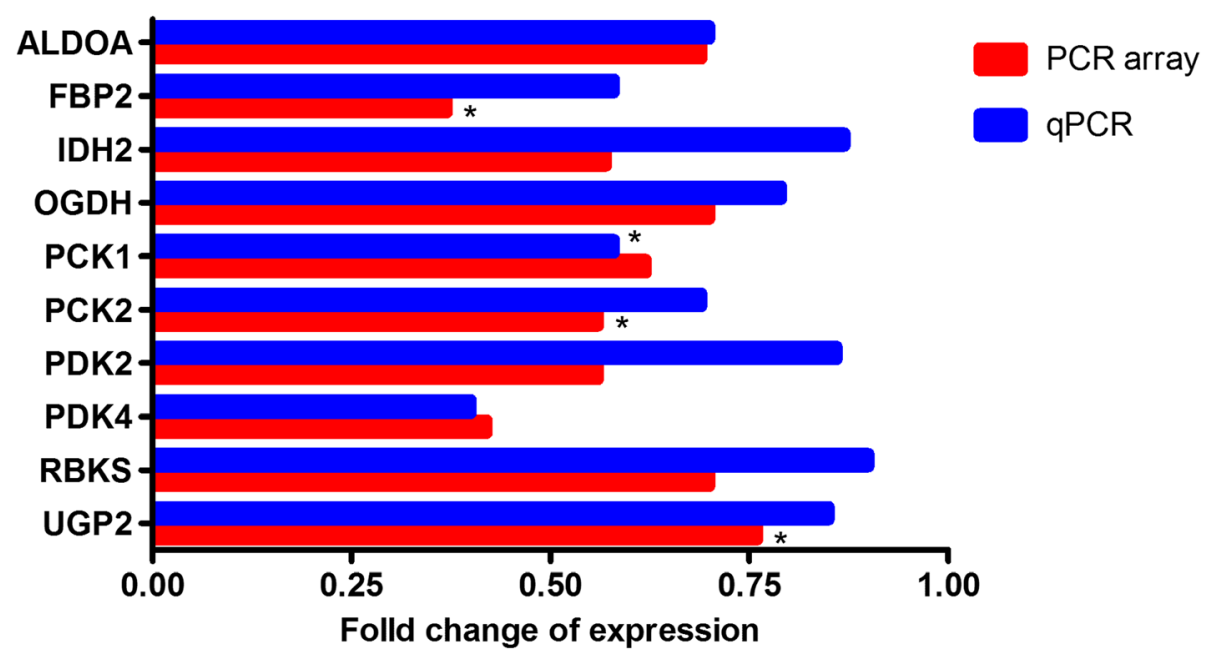

Fig. 4 Fold changes of the candidate genes expressions between oil tea and control groups by using $\mathrm{RT}^{2}$ profiler PCR array and validated with qPCR. Differences were assessed by t-test or Wilcoxon rank-sum test, as appropriate. *Significantly different from the control group on gene expression $\left({ }^{*} p<0.05\right)$ 

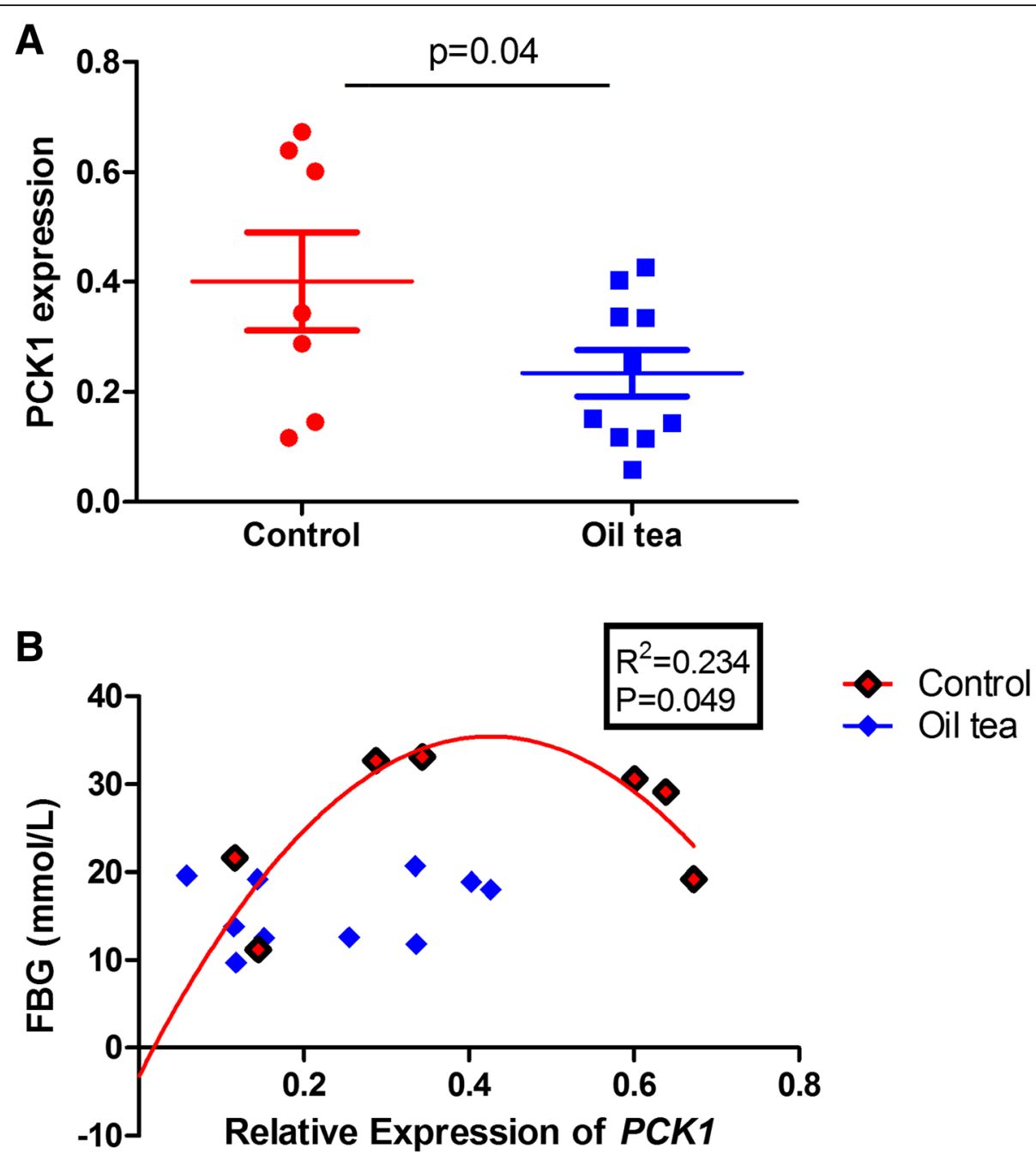

Fig. 5 PCK1 expression responded to oil tea treatment. a Decreased hepatic $P C K 1$ expression in oil tea treatment group. $\mathbf{b}$ Correlation between PCK1 expression and fasting glucose levels. FBG, fasting blood glucose; control group $(n=7)$; oil tea group $(n=10)$. Values are presented as means \pm SEM. Differences were assessed by t-test. Correlation analysis was performed with Pearson pairwise test

biological process, including six types of metabolic process (hexose, monosaccharide, glucose, carbohydrate and pyruvate metabolic process), gluconeogenesis and three types of biosynthetic process. (Fig. 6b, Additional file 1: Table S1).

\section{General characteristics of the human subjects in the case-control analysis}

A total of 372 subjects were recruited in the minority areas of Guangxi, China, including 86 T2D cases and 285 healthy controls. The clinical characteristics and biochemical indexes between T2D patients and controls are shown in Table 1 . Significant differences were observed in age, sex, BMI, FBG, LDL, TC, TG and anemia between the two groups, all higher among the cases than controls.
The association between the PCK1 SNPs and type 2 diabetes The three tested SNPs were in Hardy-Weinberg equilibrium with $p>0.05$. As shown in the Table 2, two SNPs (rs707555 and rs2071023) were significantly associated with T2D, persisting after adjustment for age, sex, BMI, family history of diabetes, TC and TG $\left(\mathrm{P}_{\text {trend }}=0.002\right.$ and $\mathrm{P}_{\text {trend }}=0.02$, respectively). A clear dose response was observed with the two SNPs as well, such that compared to those non-carrying $\mathrm{G}$ allele of rs707555, the subjects carrying one or two of $\mathrm{G}$ alleles decreased $62 \%$ (OR = $0.38, p=0.002)$ and $82 \%(\mathrm{OR}=0.18, p=0.08)$ risk of T2D, respectively. As for rs2071023, those subjects carrying one or two of $\mathrm{G}$ alleles increased 1.94 times $(p=0.03)$ and 2.39 times $(p=0.07)$ risk of T2D, respectively. Meanwhile, a significant negative correlation between rs707555 and FBG level was observed after adjustment for age, sex, BMI, family history of 


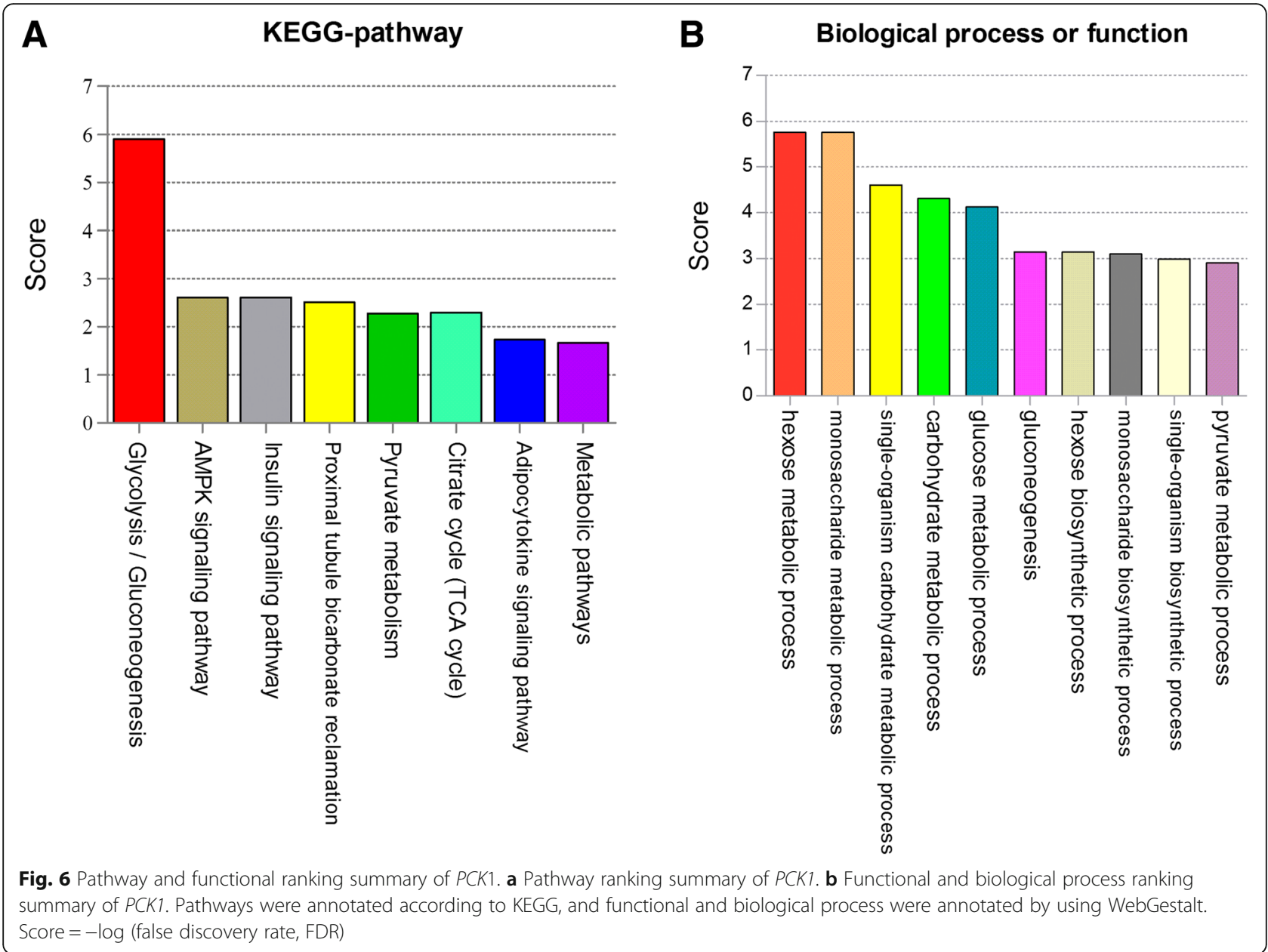

diabetes, TC and TG $\left(\mathrm{P}_{\text {trend }}=0.01\right)$, as well as a significant positive correlation between rs2071023 and TG level after adjustment for age, sex, BMI and TC $\left(P_{\text {trend }}=0.027\right)$. However, no significance was observed between the correlation of 2,071,023 and FBG, rs707555 and TG. No significant associations were observed between rs2070755 and T2D, FBG or TG (Additional file 1: Table S2).

\section{Discussion}

In the present study, we have shown that oil tea treatment can impact upon glucose homeostasis in type 2 diabetic

Table 1 The main phenotypic characteristics of the study participants

\begin{tabular}{llll}
\hline & Controls $(n=285)$ & T2D Patients $(n=86)$ & $p$-value \\
\hline Sex (male/female) & $89 / 196$ & $45 / 41$ & $<0.001$ \\
Age (years) & $53.56 \pm 16.33$ & $62.62 \pm 11.90$ & $<0.001$ \\
BMI $\left(\mathrm{Kg} / \mathrm{m}^{2}\right)$ & $22.57 \pm 3.60$ & $23.96 \pm 3.24$ & 0.003 \\
TG $(\mathrm{mmol} / \mathrm{L})$ & $1.45 \pm 0.84$ & $2.03 \pm 1.67$ & $<0.001$ \\
TC $(\mathrm{mmol} / \mathrm{L})$ & $4.69 \pm 1.10$ & $5.26 \pm 1.02$ & $<0.001$ \\
FBG $(\mathrm{mmol} / \mathrm{L})$ & $4.86 \pm 0.64$ & $8.57 \pm 4.02$ & $<0.001$ \\
HDL-cholesterol $(\mathrm{mmol} / \mathrm{L})$ & $1.77 \pm 0.46$ & $1.72 \pm 0.57$ & 0.4025 \\
LDL-cholesterol $(\mathrm{mmol} / \mathrm{L})$ & $2.27 \pm 0.80$ & $2.62 \pm 0.80$ & 0.0004 \\
Anemia $(n, \%)$ & $36(12.9 \%)$ & $35(40.7 \%)$ & $<0.001$ \\
Family history of diabetes $(n, \%)$ & $17(5.96 \%)$ & $3(3.49 \%)$ & 0.373 \\
\hline
\end{tabular}

$B M I$ body mass index, $T G$ triglyceride, $T C$ total cholesterol, $F B G$ fasting blood glucose, $H D L$ high density lipoprotein, $L D L$ low density lipoprotein 


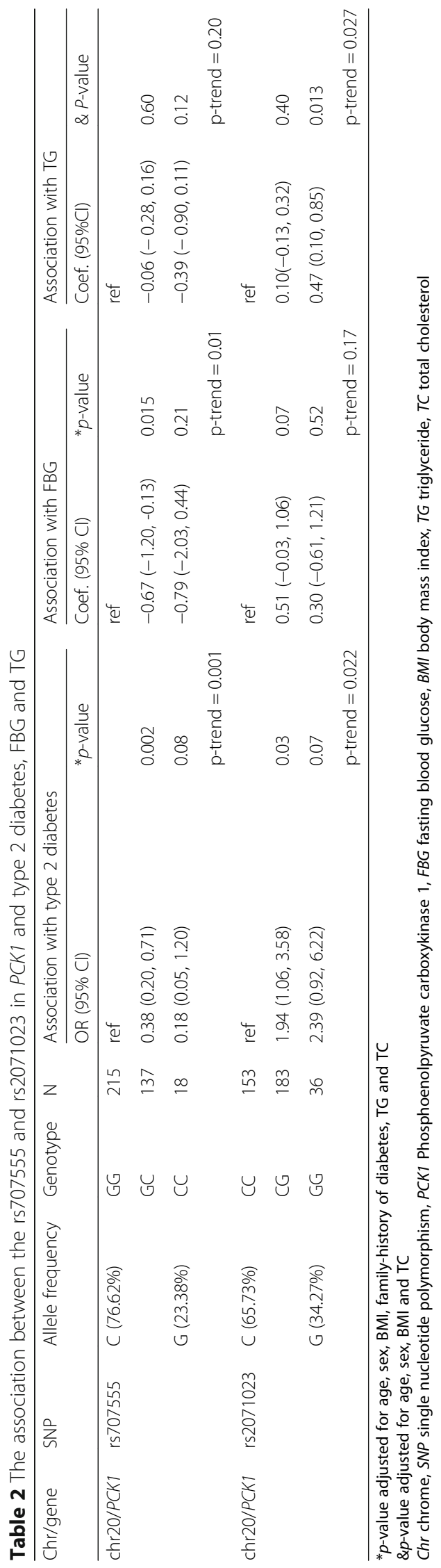


mice, as shown by decreased fasting blood glucose and total cholesterol, and improved glucose tolerance. We also identified suppression of hepatic PCK1 in oil tea-treated $\mathrm{db} / \mathrm{db}$ mice, and PCK1 expression significantly correlated with the level of fasting blood glucose. PCK1 was mainly involved in the glycolysis/gluconeogenesis pathway, and two SNPs (rs707555 and rs2071023) in PCK1 were significantly associated with T2D in the minority population in Guangxi, China.

Oil tea is a type of traditional tea beverage traditionally used in the minority areas in Guangxi Province, China for prevention and treatment of multiple ailments [2]. The main ingredients of oil tea - green tea and ginger, are well-known Chinese Materia Medica [23], having been traditionally used in traditional Chinese medicine and Southeast Asian system medicine [4, 24]. Recent evidence has shown green tea and ginger individually exert antidiabetic effects, including in animal experiments [5, 25-27], cohort studies [28, 29], and randomized controlled trials $[3,30]$. As a complex mixture of green tea, ginger and oil, we previously showed that oil tea improved hyperglycemia, glucose intolerance and hyperlipidemia in $\mathrm{db} / \mathrm{db}$ mice [1]. In the present study, the similar beneficial effects on anti-diabetes of oil tea were observed as well.

To explore the underlying mechanisms of oil tea induced antidiabetic effects, the expression of 84 genes involved in glucose metabolism were examined. Five candidate genes were found with expression changed following oil tea treatment, and they were mainly involved in the glycolysis/gluconeogenesis pathway and participated in different types of metabolism process including glucose metabolic process and gluconeogenesis. It is well established that the rate of gluconeogenesis is modulated by several enzymes, including PCK, fructose-1, 6-biphosphatase, and glucose-6-phosphatase [31-33], in which PCK1 catalyzes the first committed step [34]. In this study, we found that PCK1 expression was significantly down-regulated after oil tea treatment, and this down-regulation was positive correlated with fasting glucose level in $\mathrm{db} / \mathrm{db}$ mice. This indicated the observed decreased glucose level may be due to a lower gluconeogenesis rate due to decreased expression of PCK1. PCK1 is mainly involved in glycolysis/gluconeogenesis pathways, as well as glucose related-biological process which are involved in glucose homeostasis and type 2 diabetes $[35,36]$. These suggest PCK1 may act via glycolysis/gluconeogenesis pathways and thus affect diabetes status during oil tea treatment. Notably, as the first-line medication for treating T2D, MET was designed as the positive control in the animal experiment. It is interesting to find that oil tea showed a cumulative effect on glucose control over days/weeks, while MET affected glucose level much earlier. This indicate MET knocks the gluconeogenesis pathway on the head, while oil tea works in a slower route, for reducing expression of PCK1 and as it accumulates over days/weeks, glucose levels reduce.

Supporting these results, two SNPs (the missense rs707555 and the promoter rs2071023) in PCK1 were found to be associated with T2D in minority population in Guangxi, which has a high consumption of oil tea. Previously, research in the Eastern Chinese population showed both of these SNPs were associated with the risk of T2D [15]. Although a different allelic frequency of rs707555 was observed in the minority population of Guangxi, the association between the two SNPs and T2D were consistent with that of in the Eastern Chinese population. Furthermore, we also found that those subjects carrying CG genotype of rs707555 had significantly lower FBG, and people carrying GG genotype of rs2071023 were significantly correlated with higher TG. These findings further supported the association between the two SNPs and T2D since FBG and TG are the key biochemical markers of T2D. Taken together, these results suggest that $P C K 1$ is a genetic marker of T2D in the Chinese minority population, and thus, interventions which decrease PCK1 gene expression, such as oil tea consumption, may be effective for T2D prevention or treatment. However, such beneficial effects of oil tea requires investigation in randomized controlled trials.

\section{Conclusions}

Overall, we found that oil tea improved fasting and postprandial glucose and total cholesterol. We have identified a potential molecular mechanism underlying oil tea induced-antidiabetic effects through which expression of PCK1 was reduced. Our findings suggest PCK1 plays a key role in the beneficial effects of oil tea, and PCK1 may be a potential genetic marker for the treatment of type 2 diabetes. Randomized controlled trials to substantiate the clinical efficacy of oil tea in $\mathrm{T} 2 \mathrm{D}$ are required.

\section{Additional file}

Additional file 1: Table S1. The enriched terms of differential expression genes by using WebGestalt. Table S2. The association between the rs20707555 in PCK1 and type 2 diabetes, FBG and TG. (DOC $68 \mathrm{~kb})$

\section{Abbreviations}

AUC: area under the curve; BW: body weight; CT: cycle threshold; FBG: fasting blood glucose; FDR: false discovery rate; HWE: Hardy-Weinberg equilibrium; KEGG: Kyoto Encyclopedia of Genes and Genomes;

MET: metformin; OGTT: oral glucose tolerance test;

PCK1: Phosphoenolpyruvate carboxykinase 1; PCR: polymerase chain reaction: qPCR: quantitative PCR; RT: real-time; SNP: single nucleotide polymorphism; T2D: type 2 diabetes; TC: total cholesterol; TG: triglycerides; WebGestalt: WEBbased GEne SeT AnaLysis Toolkit 


\section{Acknowledgements}

Not applicable.

\section{Funding}

This work was supported by the National Natural Science Foundation of China (No.81660540), Natural Science Foundation of Guangxi Province (Grant No. 2015GXNSFCA139010) and the Hundred Talents Program of Guangxi universities (No. GuiliaoRen [2016] 56).

\section{Availability of data and materials}

The datasets used during the present study are available from the corresponding author upon reasonable request.

\section{Authors' contributions}

Conceptualization, $\mathrm{HZ}$ and RL; Data curation, HC; Formal analysis, $\mathrm{OH}$ and $\mathrm{YZ}$; Investigation, $\mathrm{HC}, \mathrm{QH}, \mathrm{XH}, \mathrm{WH}$ and HY; Supervision, RL; Writing - original draft, RL; Writing - review \& editing, SS. All authors read and approved the final manuscript.

\section{Ethics approval}

The animal experimental protocols used in this study were approved by the Animal Ethics Committee of Guangxi Center for Disease Prevention and Control (No. 20160003). The protocol of population study was approved by the Ethics Committee of Guangxi Center for Disease Prevention and Control (No.GXIRB2016-0014), all the participants signed informed consent.

\section{Consent for publication}

Not applicable.

\section{Competing interests}

The authors declare that they have no competing interests.

\section{Publisher's Note}

Springer Nature remains neutral with regard to jurisdictional claims in published maps and institutional affiliations.

\section{Author details}

${ }^{1}$ Center for Genomic and Personalized Medicine, Guangxi Medical University, 22 Shuangyong Road, Nanning 530021, Guangxi, China. ${ }^{2}$ Guangxi Center for Disease Prevention and Control, Nanning, China. ${ }^{3}$ General Practice School, Guangxi Medical University, Nanning, China. ${ }^{4}$ Melbourne School of Population \& Global Health, University of Melbourne, Carlton, Australia. ${ }^{5}$ Menzies Institute for Medical Research, University of Tasmania, Hobart, Australia. ${ }^{6}$ Public Health School, Guangxi Medical University, 22 Shuangyong Road, Nanning 530021, Guangxi, China.

Received: 9 November 2018 Accepted: 24 January 2019

Published online: 13 February 2019

\section{References}

1. Lin R, He X, Chen H, He Q, Yao Z, Li Y, Yang H, Simpson S Jr. Oil tea improves glucose and lipid levels and alters gut microbiota in type 2 diabetic mice. Nutr Res. 2018;57:67-77.

2. Hong $X$. Yao tea and disease preventiona and ailment treatment. J Med Pharm Chin Minorities. 2015:21:75-6.

3. Liu K, Zhou R, Wang B, Chen K, Shi LY, Zhu JD, Mi MT. Effect of green tea on glucose control and insulin sensitivity: a meta-analysis of 17 randomized controlled trials. Am J Clin Nutr. 2013;98:340-8.

4. Bode AM, Dong Z. The amazing and mighty ginger. In herbal medicine: biomolecular and clinical aspects. 2nd edition. Edited by Benzie IFF, Wachtel-Galor S. Boca Raton: CRC Press/Taylor \& Francis; 2011.

5. Akash MS, Rehman K, Tariq M, Chen S. Zingiber officinale and type 2 diabetes mellitus: evidence from experimental studies. Crit Rev Eukaryot Gene Expr. 2015:25:91-112.

6. Hanson RW, Reshef L. Regulation of phosphoenolpyruvate carboxykinase (GTP) gene expression. Annu Rev Biochem. 1997;66:581-611.

7. Clouthier DE, Comerford SA, Hammer RE. Hepatic fibrosis, glomerulosclerosis, and a lipodystrophy-like syndrome in PEPCK-TGF-beta1 transgenic mice. J Clin Invest. 1997;100:2697-713.

8. Sun Y, Liu S, Ferguson S, Wang L, Klepcyk P, Yun JS, Friedman JE. Phosphoenolpyruvate carboxykinase overexpression selectively attenuates insulin signaling and hepatic insulin sensitivity in transgenic mice. J Biol Chem. 2002:277:23301-7.

9. Valera A, Pujol A, Pelegrin M, Bosch F. Transgenic mice overexpressing phosphoenolpyruvate carboxykinase develop non-insulin-dependent diabetes mellitus. Proc Natl Acad Sci U S A. 1994;91:9151-4.

10. Gomez-Valades AG, Mendez-Lucas A, Vidal-Alabro A, Blasco FX, Chillon M, Bartrons R, Bermudez J, Perales JC. Pck1 gene silencing in the liver improves glycemia control, insulin sensitivity, and dyslipidemia in $\mathrm{db} / \mathrm{db}$ mice. Diabetes. 2008;57:2199-210.

11. Runtuwene J, Cheng KC, Asakawa A, Amitani H, Amitani M, Morinaga A, Takimoto Y, Kairupan BH, Inui A. Rosmarinic acid ameliorates hyperglycemia and insulin sensitivity in diabetic rats, potentially by modulating the expression of PEPCK and GLUT4. Drug Des Devel Ther. 2016;10:2193-202.

12. Li Y, Liu Y, Chen G. Vitamin a status affects the plasma parameters and regulation of hepatic genes in streptozotocin-induced diabetic rats. Biochimie. 2017;137:1-11.

13. Oda H, Okuda Y, Yoshida Y, Kimura N, Kakinuma A. Phenobarbital reduces blood glucose and gluconeogenesis through down-regulation of phosphoenolpyruvate carboxykinase (GTP) gene expression in rats. Biochem Biophys Res Commun. 2015;466:306-11.

14. Beale EG, Harvey BJ, Forest C. PCK1 and PCK2 as candidate diabetes and obesity genes. Cell Biochem Biophys. 2007;48:89-95.

15. Dong Y, Zhang H, Wang X, Feng Q, Chen X, Su Q. A Leu184Val polymorphism in PCK1 gene is associated with type 2 diabetes in eastern Chinese population with $B M 1<23$ kg/m2. Diabetes Res Clin Pract. 2009;83:227-32.

16. Rees SD, Britten AC, Bellary S, O'Hare JP, Kumar S, Barnett AH, Kelly MA. The promoter polymorphism $-232 \mathrm{C} / \mathrm{G}$ of the PCK1 gene is associated with type 2 diabetes in a UK-resident south Asian population. BMC Med Genet. 2009;10:83.

17. Willer CJ, Bonnycastle LL, Conneely KN, Duren WL, Jackson AU, Scott LJ, Narisu N, Chines PS, Skol A, Stringham HM, et al. Screening of 134 single nucleotide polymorphisms (SNPs) previously associated with type 2 diabetes replicates association with 12 SNPs in nine genes. Diabetes. 2007:56:256-64.

18. Hou X, Hao E, Fan L, Du Z, Xie H, Qin J, Xie J, Deng J. Study on the scientific connotation and industrial development of oil tea in Gongcheng Yao Autonomous County, Guangxi. J Med Pharm Chin Minorities. 2017;7:1-4.

19. Livak KJ, Schmittgen TD. Analysis of relative gene expression data using real-time quantitative PCR and the 2(-Delta Delta C(T)) method. Methods. 2001:25:402-8.

20. Department of Noncommunicable Disease Surveillance. Definition, diagnosis and classification of diabetes mellitus and its complications: report of a WHO consultation. Part 1. Diagnosis and classification of diabetes mellitus. Geneva: World Health Organization; 1999.

21. Liu Z, Yang Z, Jiang L, Gao H. The effect of Youfuquan water on type 2 diabetic rats. Med J Qilu. 2015;30:677-80.

22. Purcell S, Neale B, Todd-Brown K, Thomas L, Ferreira MA, Bender D, Maller J, Sklar P, de Bakker PI, Daly MJ, Sham PC. PLINK: a tool set for whole-genome association and population-based linkage analyses. Am J Hum Genet. 2007; 81:559-75.

23. Li S. Compendium of Materia Medica. Beijing: China Press of Traditional Chinese Medicine; 2013.

24. Serafini M, Del Rio D, Yao DN, Bettuzzi S, Peluso I. Health Benefits of Tea. In: IFF B, Wachtel-Galor S, editors. Herbal Medicine: Biomolecular and Clinical Aspects. 2nd edition. Boca Raton (FL): CRC Press/Taylor \& Francis; 2011.

25. Ortsater H, Grankvist N, Wolfram S, Kuehn N, Sjoholm A. Diet supplementation with green tea extract epigallocatechin gallate prevents progression to glucose intolerance in $\mathrm{db} / \mathrm{db}$ mice. Nutr Metab (Lond). 2012:9:11.

26. Wein S, Schrader E, Rimbach G, Wolffram S. Oral green tea catechins transiently lower plasma glucose concentrations in female $\mathrm{db} / \mathrm{db}$ mice. J Med Food. 2013;16:312-7.

27. Bae KC, Park JH, Na AY, Kim SJ, Ahn S, Kim SP, Oh BC, Cho HC, Kim YW, Song DK. Effect of green tea extract/poly-gamma-glutamic acid complex in obese type 2 diabetic mice. Diabetes Metab J. 2013;37:196-206.

28. Yang WS, Wang WY, Fan WY, Deng Q, Wang $X$. Tea consumption and risk of type 2 diabetes: a dose-response meta-analysis of cohort studies. Br J Nutr. 2014:111:1329-39.

29. Jing $Y$, Han G, Hu Y, Bi Y, Li L, Zhu D. Tea consumption and risk of type 2 diabetes: a meta-analysis of cohort studies. J Gen Intern Med. 2009;24:557-62. 
30. Mozaffari-Khosravi H, Talaei B, Jalali BA, Najarzadeh A, Mozayan MR. The effect of ginger powder supplementation on insulin resistance and glycemic indices in patients with type 2 diabetes: a randomized, doubleblind, placebo-controlled trial. Complement Ther Med. 2014;22:9-16.

31. Surwit RS, Kuhn CM, Cochrane C, McCubbin JA, Feinglos MN. Diet-induced type II diabetes in C57BL/6J mice. Diabetes. 1988;37:1163-7.

32. Vidal-Puig A, O'Rahilly S. Metabolism. Controlling the glucose factory. Nature. 2001;413:125-6.

33. Shao J, Qiao L, Janssen RC, Pagliassotti M, Friedman JE. Chronic hyperglycemia enhances PEPCK gene expression and hepatocellular glucose production via elevated liver activating protein/liver inhibitory protein ratio. Diabetes. 2005;54:976-84.

34. Quinn PG, Yeagley D. Insulin regulation of PEPCK gene expression: a model for rapid and reversible modulation. Curr Drug Targets Immune Endocr Metabol Disord. 2005:5:423-37.

35. Oh KJ, Han HS, Kim MJ, Koo SH. Transcriptional regulators of hepatic gluconeogenesis. Arch Pharm Res. 2013;36:189-200.

36. Desjardins EM, Steinberg GR. Emerging role of AMPK in Brown and Beige adipose tissue (BAT): implications for obesity, insulin resistance, and type 2 diabetes. Curr Diab Rep. 2018;18:80

Ready to submit your research? Choose BMC and benefit from:

- fast, convenient online submission

- thorough peer review by experienced researchers in your field

- rapid publication on acceptance

- support for research data, including large and complex data types

- gold Open Access which fosters wider collaboration and increased citations

- maximum visibility for your research: over $100 \mathrm{M}$ website views per year

At BMC, research is always in progress.

Learn more biomedcentral.com/submissions 